\title{
Comparative Analysis of Varios Diversity Techniques for Ofdm Systems
}

\author{
Reena ${ }^{1}$ M.Tech., Sahasha Namdeo ${ }^{2}$ M.Tech. \\ (Maharishi Dayanand University, Rohtak, India)
}

\begin{abstract}
In this paper, three transmit diversity techniques are proposed that use extra transmit antennas to obtain additional diversity. An analytical expression for the signal-to-noise ratio (SNR) and bit-error-rate at the output of a three-branch maximal ratio combining, equal gain combining and selection diversity system is given. The three branches are assumed to be Rayleigh fading, correlated with the BPSK modulation. Measurements of the signal-to-noise ratio and bit-error-rate after selection, equal gain combining and maximal ratio combining were made in Rayleigh fading channels and compared with the analytical results. Also presented are the exact analytical average probabilities of bit error for coherent binary phase-shift keying for three-branch maximal ratio combining, equal gain combining and selective diversity for Rayleigh fading channel. All these three branches is compared on the basis of signal to noise ratio and bit error rate with the increasing no. of receiver. This work confirms the benefits of choosing the maximal ratio combining instead of equal gain combining and selection diversity by measuring the performances of these three branches for SNR and BER.
\end{abstract}

Keywords - BPSK, EGC, LOS, MRC, PDF, RF, SNR,BER

\section{Introduction}

Antenna diversity, also known as space diversity, is any one of several wireless diversity schemes that uses two or more antennas to improve the quality and reliability of a wireless link. Often, especially in urban and indoor environments, there is no clear line-of-sight (LOS) between transmitter and receiver. Instead the signal is reflected along multiple paths before finally being received. Each of these bounces can introduce phase shifts, time delays, attenuations, and distortions that can destructively interfere with one another at the aperture of the receiving antenna. In most scattering environments, antenna diversity is a practical, effective and, hence, a widely applied technique for reducing the effect of multipath fading [1].

Antenna diversity is especially effective at mitigating these multipath situations. This is because multiple antennas offer a receiver several observations of the same signal. Each antenna will experience a different interference environment. Thus, if one antenna is experiencing a deep fade, it is likely that another has a sufficient signal. Collectively such a system can provide a robust link. While this is primarily seen in receiving systems (diversity reception), the analog has also proven valuable for transmitting systems (transmit diversity) as well.

The classical approach is to use multiple antennas at the receiver and perform combining or selection and switching in order to improve the quality of received signal. The problem with this system is the cost, size and power of the remote units. The use of multiple antennas and RF chains (selections and switching circuits) makes the remote units larger and more expensive. As a result, diversity techniques have almost exclusively applied to base stations to improve their reception quality. Base station often serves hundreds to thousands of remote units. It is economical to add equipments to base stations rather than the remote units. For this reason, transmit diversity schemes are very attractive. For instance, one antenna and one transmit chain may be added to base station to improve the reception quality of all the remote units in that the station's coverage area [2].

Inherently an antenna diversity scheme requires additional hardware and integration versus a single antenna system but due to the commonality of the signal paths a fair amount of circuitry can be shared. Also with the multiple signals there is a greater processing demand placed on the receiver, which can lead to tighter design requirements. Typically, however, signal reliability is paramount and using multiple antennas is an effective way to decrease the number of drop-outs and lost connections.

Diversity techniques are used to improve the performance of the radio channel without any increase in the transmitted power[5].

\section{Selection Diversity}

Selection Processing presents only one antenna's signal to the receiver at any given time [5]. The antenna chooses the best SNR among the received signals. In the figure 1 shown below the receiver selects the signal having max SNR. 


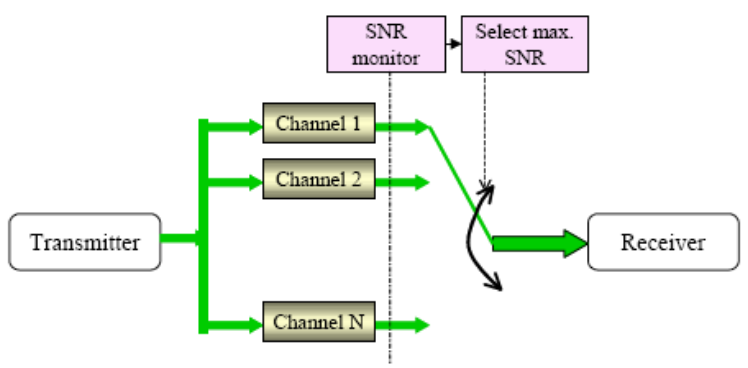

Fig 1.Selection Diversity

In selection diversity, we will assume that the channel is a flat fading Rayleigh multipath channel and the modulation is BPSK.

1. We have $\mathrm{N}$ receive antennas and one transmit antenna.

2. The channel is flat fading - In simple terms, it means that the multipath channel has only one tap. So, the convolution operation reduces to a simple multiplication.

3. The channel experienced by each receive antenna is randomly varying in time. For any receiving antenna, each transmitted symbol gets multiplied by a randomly varying complex number $\mathrm{h}_{\mathrm{i}}$. As the channel under consideration is a Rayleigh channel, the real and imaginary parts of $h_{i}$ are Gaussian distributed having mean 0 and variance $1 / 2$.

4. The channel experience by each receive antenna is independent from the channel experienced by other receive antennas.

5. On each receive antenna, the noise $\mathrm{n}$ has the Gaussian probability density function with

$p(n)=\frac{1}{\sqrt{2 \pi \sigma^{2}}} e^{\frac{-(n-\mu)^{2}}{2 \sigma^{2}}}$ with $\mu=0$ and $\sigma^{2}=\frac{N_{0}}{2}$

The noise on each receive antenna is independent from the noise on the other receive antennas.

6. At each receive antenna, the channel $h_{i}$ is known at the receiver. For example, on any receiving antenna, equalization is performed at the receiver by dividing the received symbol $y_{i}$ by the apriori known $h_{i}$ i.e.

$\hat{y}=\frac{y_{i}}{h_{i}}=\frac{h_{i} x+n_{i}}{h_{i}}=x+\tilde{n_{i}}$

where $\quad \tilde{n_{i}}=\frac{n_{i}}{h_{i}} \quad$ is the additive noise scaled by the channel coefficient.

7. In the presence of channel $\mathrm{h}_{\mathrm{i}}$, the instantaneous bit energy to noise ratio at any receive antenna is $\frac{\left|h_{i}\right|^{2} E_{b}}{N_{0}}$. For notational convenience, let us define,

$\gamma_{i}=\frac{\left|h_{i}\right|^{2} E_{b}}{N_{0}}$

we know that, if $h_{i}$ is a Rayleigh distributed random variable, then $h_{i}{ }^{2}$ is a chi-squared random variable with two degrees of freedom. The pdf of $\gamma$ is

$p\left(\gamma_{i}\right)=\frac{1}{\left(E_{b} / N_{0}\right)} e^{\frac{-\gamma_{i}}{\left(E_{b} / N_{0}\right)}}$

\section{Equal-Gain Combining Diversity}

Various techniques are known to combine the signals from multiple diversity branches. In Equal Gain Combining, each signal branch weighted with the same factor, irrespective of the signal amplitude. However, co-phasing of all signal is needed to avoid signal cancellation[18].

Thus, EGC is simpler to implement than Maximum Ratio Combining (MRC). The adaptively controller amplifiers / attenuators are not needed. Moreover, no channel amplitude estimation is needed. 


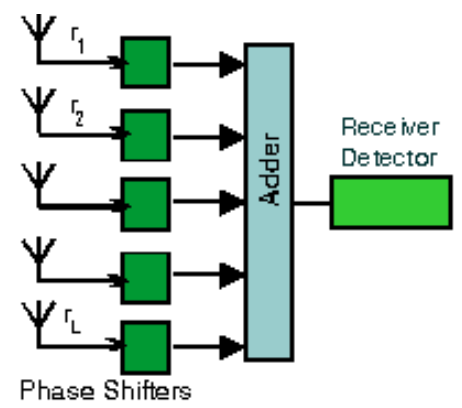

Figure: 2- branch Equal Gain Combining antenna diversity receiver $(L=5)$.

The average SNR improvement of EGC is typically about $1 \mathrm{~dB}$ worse than with MRC, but still much better than without diversity.

Receiver diversity is a form of space diversity, where there are multiple antennas at the receiver. The presence of receiver diversity poses an interesting problem - how do we use 'effectively' the information from all the antennas to demodulate the data[11].

1. We have $\mathrm{N}$ receive antennas and one transmit antenna.

2. The channel is flat fading - In simple terms, it means that the multipath channel has only one tap. So, the convolution operation reduces to a simple multiplication.

3 . The channel experienced by each receive antenna is randomly varying in time. For any receiving antenna, each transmitted symbol gets multiplied by a randomly varying complex number $h_{i}$. As the channel under consideration is a Rayleigh channel, the real and imaginary parts of $h_{i}$ are Gaussian distributed having mean $\mu_{h_{i}}=0$ and $\sigma_{h_{i}}^{2}=\frac{1}{2}$

4. The channel experience by each receive antenna is independent from the channel experienced by other receive antennas.

5. On each receive antenna, the noise $n$ has the Gaussian probability density function with

$p(n)=\frac{1}{\sqrt{2 \pi \sigma^{2}}} e^{\frac{-(n-\mu)^{2}}{2 \sigma^{2}}}$ with $\mu=0$ and $\sigma^{2}=\frac{N_{0}}{2}$

The noise on each receive antenna is independent from the noise on the other receive antennas.

6. At each receive antenna, the channel $h_{i}$ is known at the receiver.

7. In the presence of channel $h_{i}$, the instantaneous bit energy to noise ratio at any receiving antenna is $\frac{\left|h_{i}\right|^{2} E_{b}}{N_{0}}$.

For notational convenience, let us define,

$\gamma_{i}=\frac{\left|h_{i}\right|^{2} E_{b}}{N_{0}}$

we know that, if $h_{i}$ is a Rayleigh distributed random variable, then $h_{i}^{2}$ is a chi-squared random variable with two degrees of freedom. The pdf of $\gamma_{i}$ is

$p\left(\gamma_{i}\right)=\frac{1}{\left(E_{b} / N_{0}\right)} e^{\frac{-\gamma_{i}}{\left(E_{b} / N_{0}\right)}}$

\section{Maximum Ratio Combining Diversity}

Various techniques are known to combine the signals from multiple diversity branches. In Maximum Ratio combining each signal branch is multiplied by a weight factor that is proportional to the signal amplitude[11]. That is, branches with strong signal are further amplified, while weak signals are attenuated.

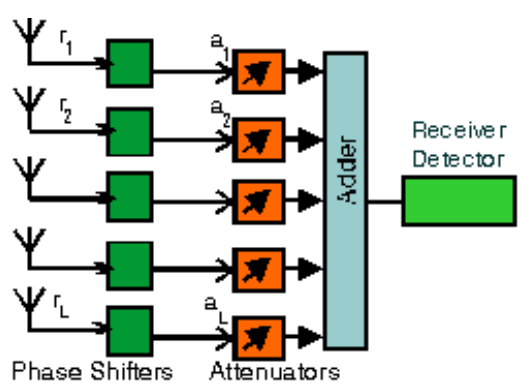

Figure: 3-branch antenna diversity receiver $(L=5)$. With MRC, the attenuation/amplification factor is proportional to the signal amplitude $a_{i}=r_{i}$ for each channel $i$. 
Receiver diversity is a form of space diversity, where there are multiple antennas at the receiver. The presence of receiver diversity poses an interesting problem - how do we use 'effectively' the information from all the antennas to demodulate the data[14].

In Maximal Ratio Combining, we will assume that the channel is a flat fading Rayleigh multipath channel and the modulation is BPSK.

We use the same constraints as defined in the Selection Diversity and Equal Gain Combining (EGC). Then repeat the same.

1. We have $\mathrm{N}$ receive antennas and one transmit antenna.

2. The channel is flat fading - In simple terms, it means that the multipath channel has only one tap. So, the convolution operation reduces to a simple multiplication.

3. The channel experienced by each receive antenna is randomly varying in time. For any receiving antenna, each transmitted symbol gets multiplied by a randomly varying complex number $h_{i}$. As the channel under consideration is a Rayleigh channel, the real and imaginary parts of $h_{i}$ are Gaussian distributed having mean $\mu_{h_{i}}=0$ and variance $\sigma_{h_{i}}^{2}=\frac{1}{2}$

4. The channel experience by each receive antenna is independent from the channel experienced by other receive antennas.

5. On each receive antenna, the noise $n$ has the Gaussian probability density function with

$p(n)=\frac{1}{\sqrt{2 \pi \sigma^{2}}} e^{-\frac{-(n-\mu)^{2}}{2 \sigma^{2}}}$ with $\mu=0$ and $\sigma^{2}=\frac{N_{0}}{2}$

The noise on each receive antenna is independent from the noise on the other receive antennas.

6. At each receive antenna, the channel $h_{i}$ is known at the receiver.

7. In the presence of channel $h_{i}$, the instantaneous bit energy to noise ratio at the receiving antenna is $\frac{\left|h_{i}\right|^{2} E_{b}}{N_{0}}$.

For notational convenience, let us define,

$\gamma_{i}=\frac{\left|h_{i}\right|^{2} E_{b}}{N_{0}}$

BER:

\section{Proposed Methodology:}

\section{V.I.I Selection Diversity}

(a) Generate random binary sequence of +1 's and -1 's.

(b) Multiply the symbols with the channel and then add white Gaussian noise.

(c) At the receiver, find the receive path with maximum power.

(d) Chose that receive path, equalize (divide) the received symbols with the known channel.

(d) Perform hard decision decoding and count the bit errors.

(e) Repeat for multiple values of $\mathrm{E}_{\mathrm{b}} / \mathrm{N}_{0}$ and plot the simulation and theoretical results.

\section{V.I.II Maximal Ratio Combining}

(a) Generate random binary sequence of +1 's and -1 's.

(b) Multiply the symbols with the channel and then add white Gaussian noise.

(c) Chose that receive path, equalize the received symbols per maximal ratio combining.

(d) Perform hard decision decoding and count the bit errors.

(e) Repeat for multiple values of $\mathrm{E}_{\mathrm{b}} / \mathrm{N}_{0}$ and plot the simulation and theoretical results.

\section{V.I.III Equal Gain Combining}

(a) Generate random binary sequence of +1 's and -1 's.

(b) Multiply the symbols with the channel and then add white Gaussian noise.

(c) At the receiver, for each receive path, equalize by compensating with the known channel phase.

(d) Accumulate the equalized symbols from all the receive paths

(d) Perform hard decision decoding and count the bit errors

(e) Repeat for multiple values of $\mathrm{E}_{\mathrm{b}} / \mathrm{N}_{0}$ and plot the simulation and theoretical results.

\section{V.I.IV Simulation Result}

In this work, we have observed the bit error rate and signal to noise ratio performances with the different receiver diversity technique, which is shown below-

\section{V.I.IV.A BER}

a) In the figure shown below the result for BER for selection diversity with Eb/No are taken. It can be clearly observed that BER is much better, when there are two receivers, which clearly shows that as the no. of receiver increases BER improves. 


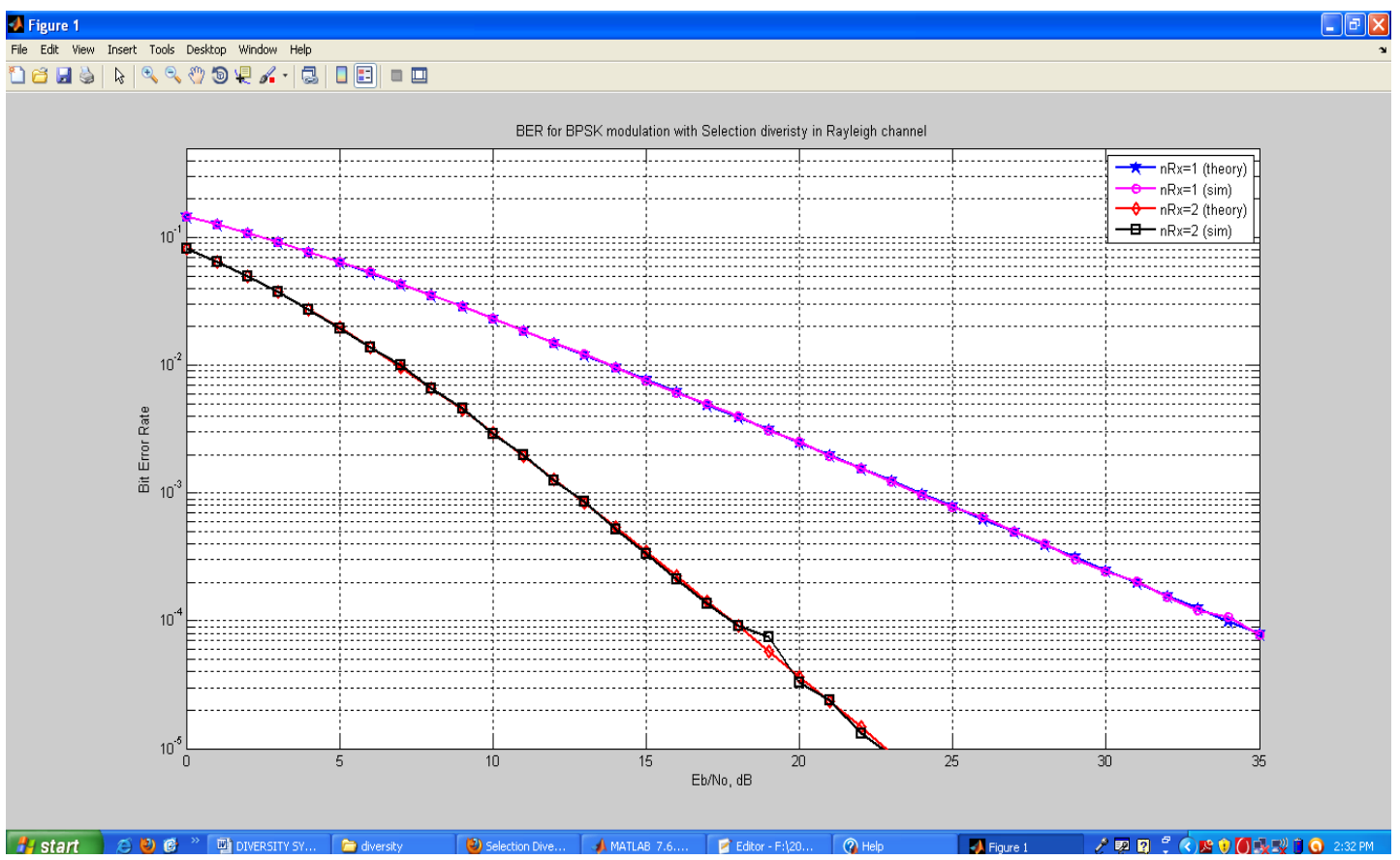

Fig: BER Vs Eb/No for selection diversity

b) In the figure shown below the result for BER for Equal Gain Combining with Eb/No are taken. It can be clearly observed that BER is much better, when there are two receivers, which clearly shows that as the no. of receiver increases BER improves.

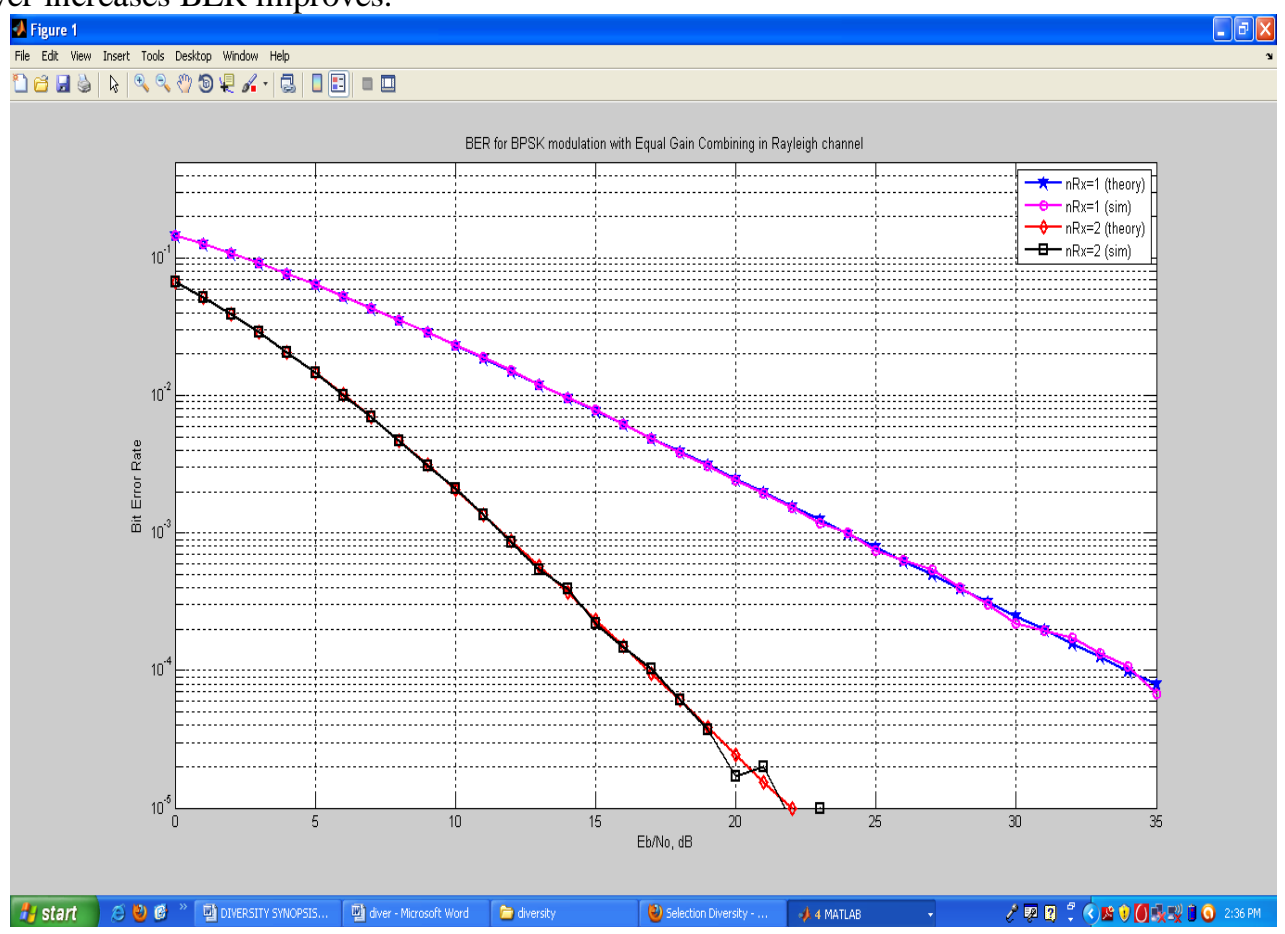

Fig: BER Vs Eb/No for Equal Gain Combinig

c) In the figure shown below the result for BER for Maximal Ratio Combining with Eb/No are taken. It can be clearly observed that BER is much better, when there are two receivers, which clearly shows that as the no. of receiver increases BER improves. 


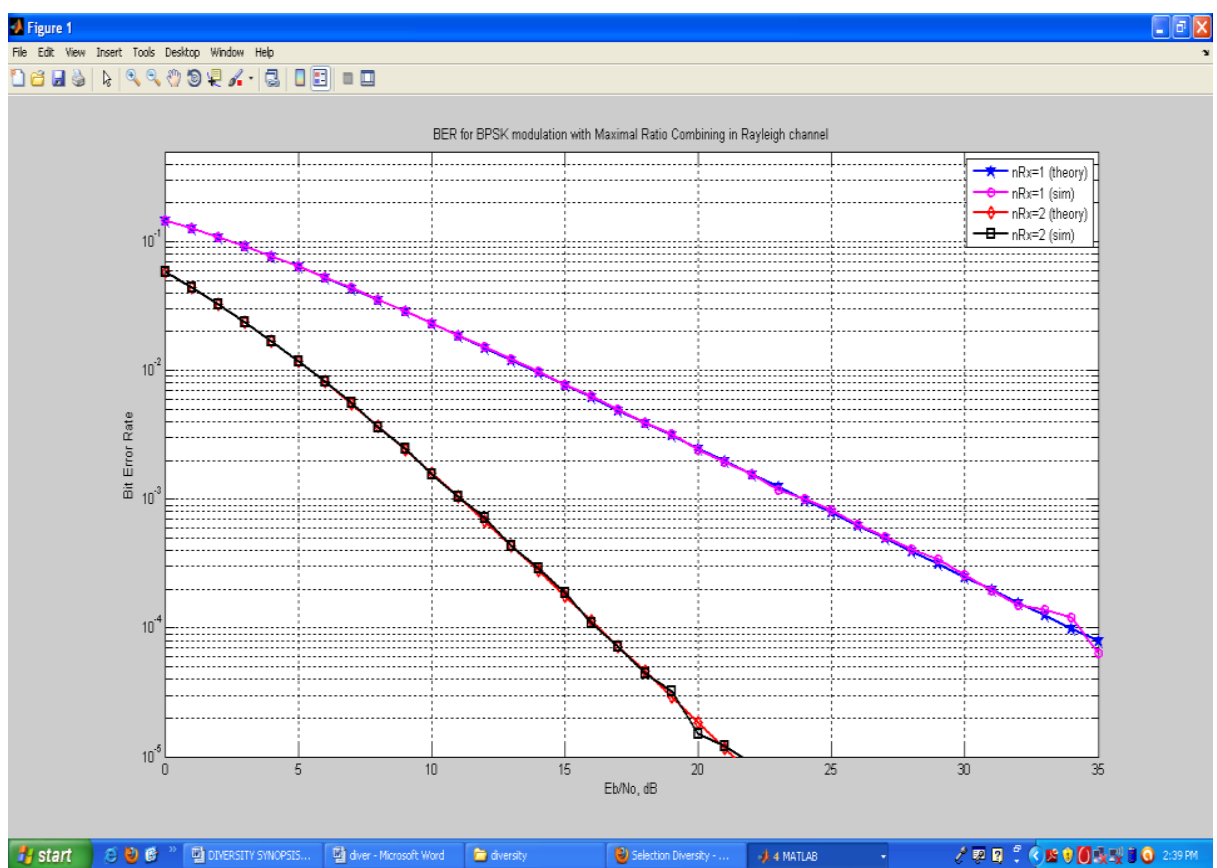

Fig: BER Vs Eb/No for Maximal Ratio Combining

\section{V.I.V Result Comparison:}

In the figure below, plot of the BER with the Eb/No for all the three techniques have been shown. This clearly shows that BER in case of MRC is much better than that of Equal Gain combining and selection diversity. If the value of Eb/No is 5 then it can be observed from the below figure the value of bit error rate is lesser for Maximal Ratio Combining diversity technique as compare to Equal Gain Combining and Selection diversity technique and BER value for Equal Gain Combining is more than the Selection diversity technique.

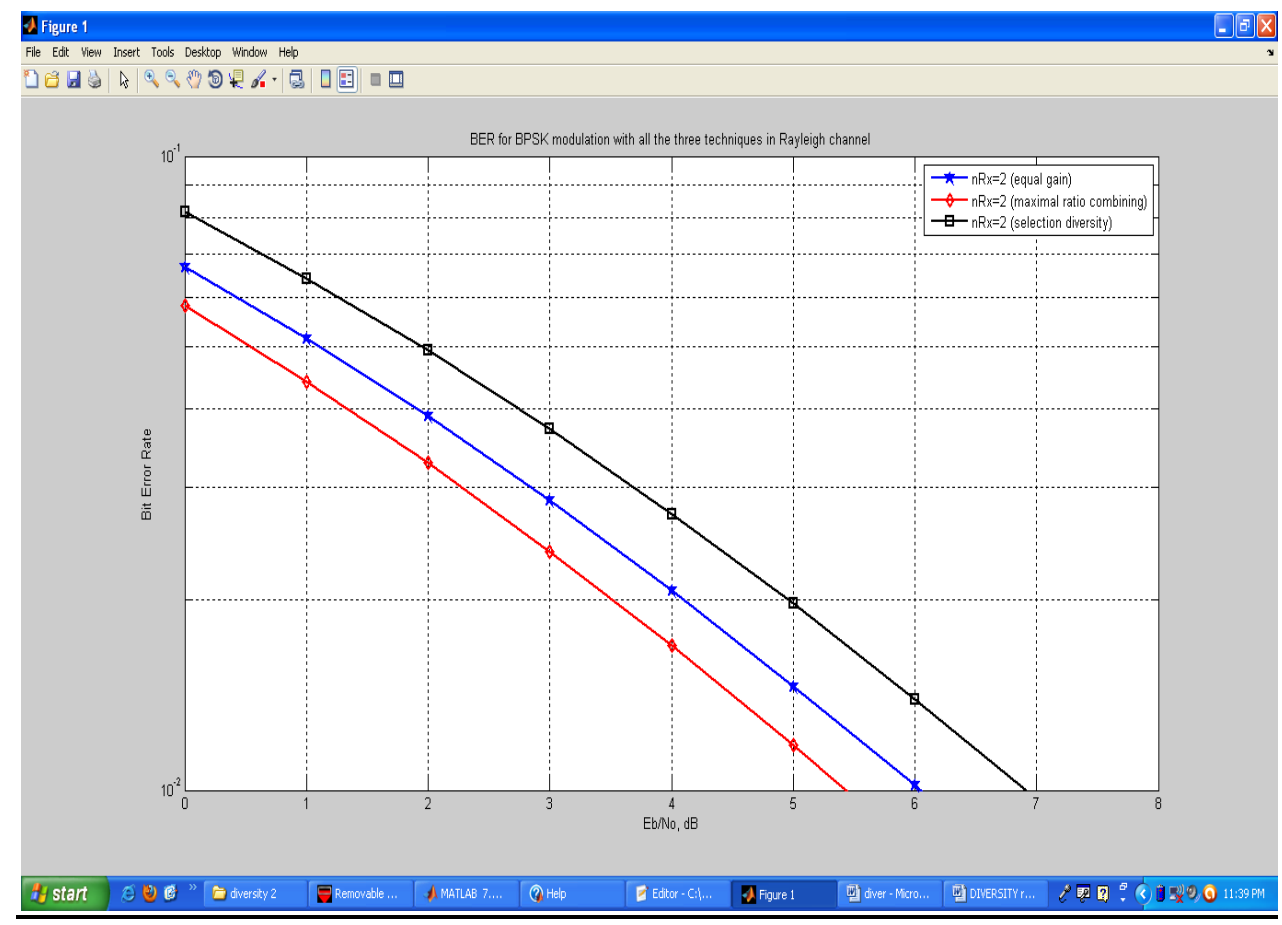

Fig: BER Comparison

\section{V.I.IV.B Signal to noise ratio}

Performance of different receiver diversity technique is shown below. In which it can be seen the changes in the value of SNR with the no. of receiving antenna. 
a) In the figure shown below the result for SNR for selection diversity with no. of receivers are taken. It can be clearly observed that SNR is much better, when no. of receiving antennas increases. which clearly shows that as the no. of receiving antenna increases SNR improves.

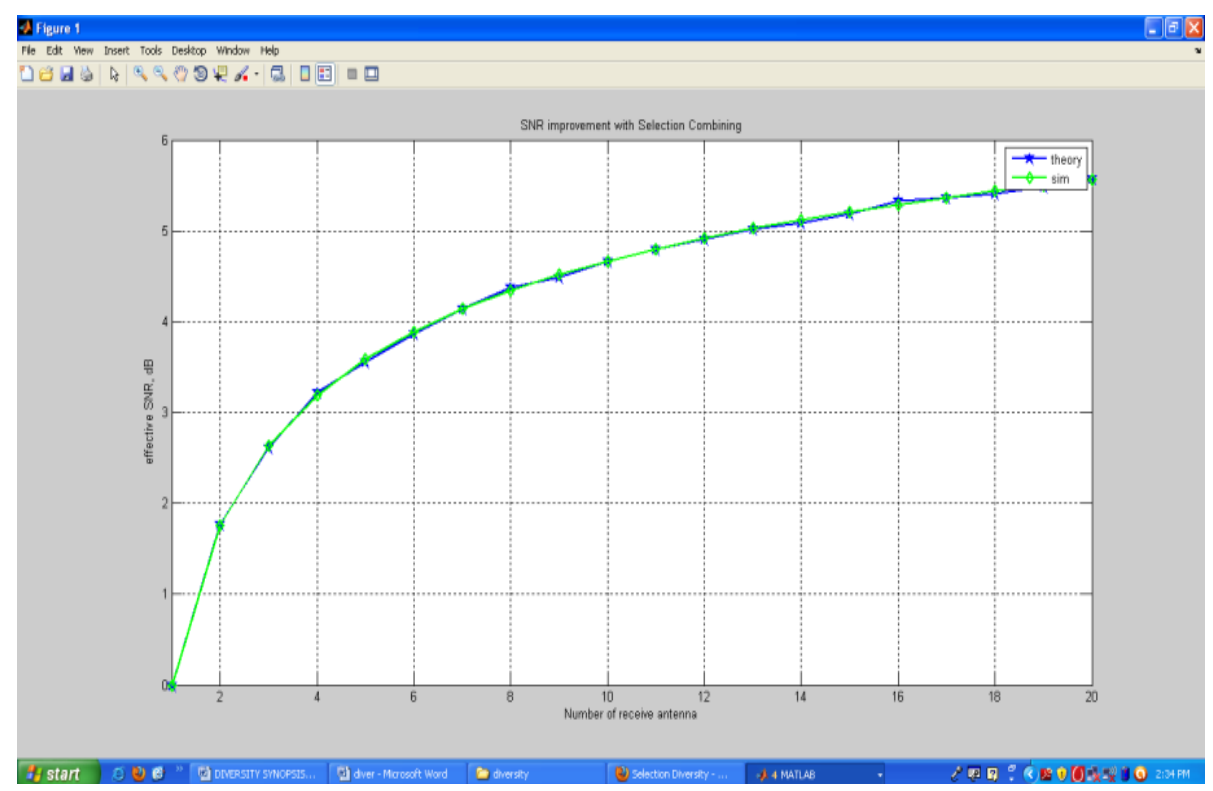

Fig: SNR for selection diversity

a) In the figure shown below the result for SNR for Equal Gain Combining with no. of receiving antenna are taken. It can be clearly observed that SNR is much better, when no. of receiving antenna increases.

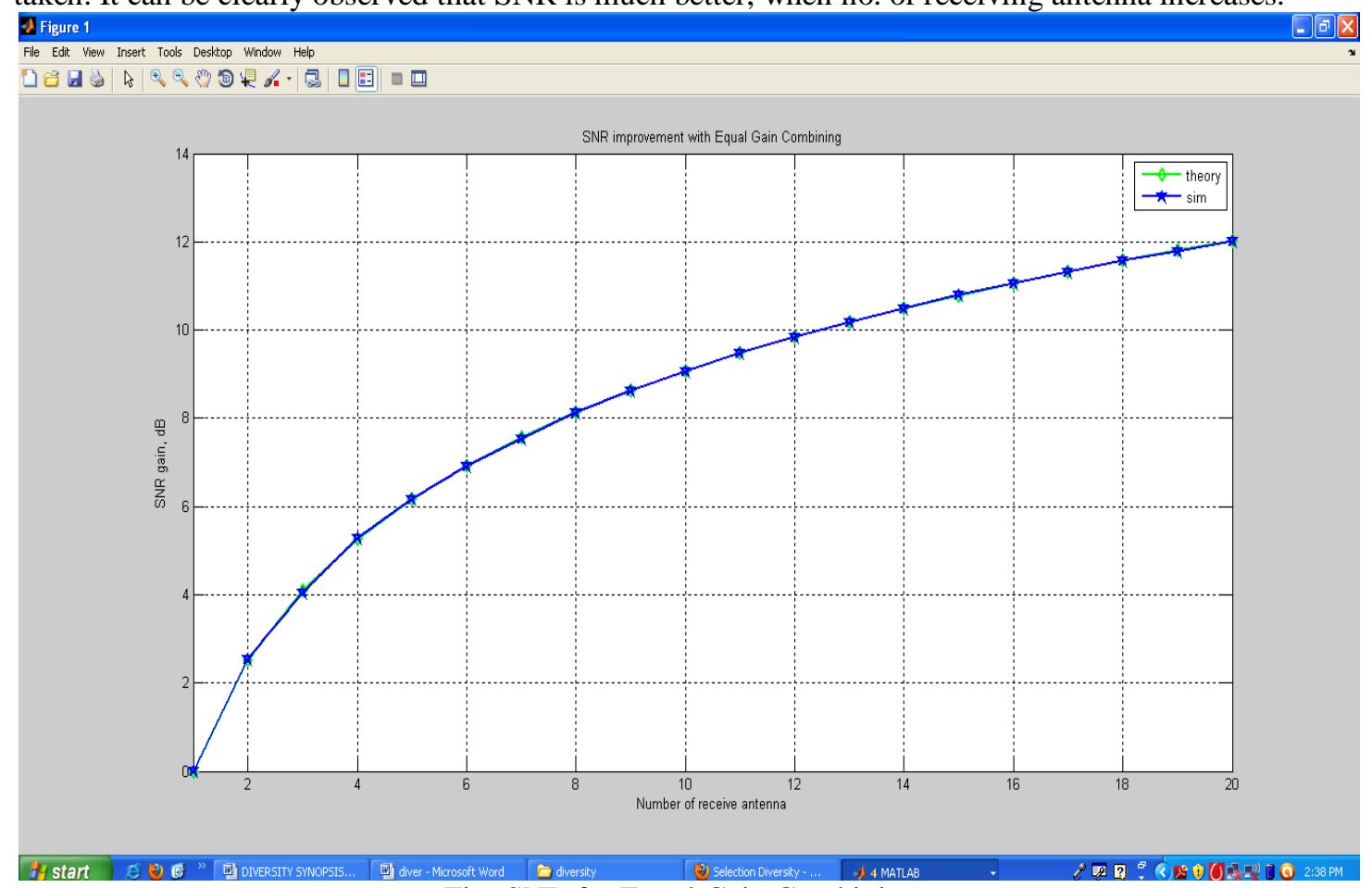

Fig: SNR for Equal Gain Combining

a) In the figure shown below the result for SNR for Maximal Ratio Combining with no. of receiving antenna are taken. It can be clearly observed that SNR is much better, when no. of receiving antenna increases. which clearly shows that as the no. of receiving antenna increases SNR improves. 


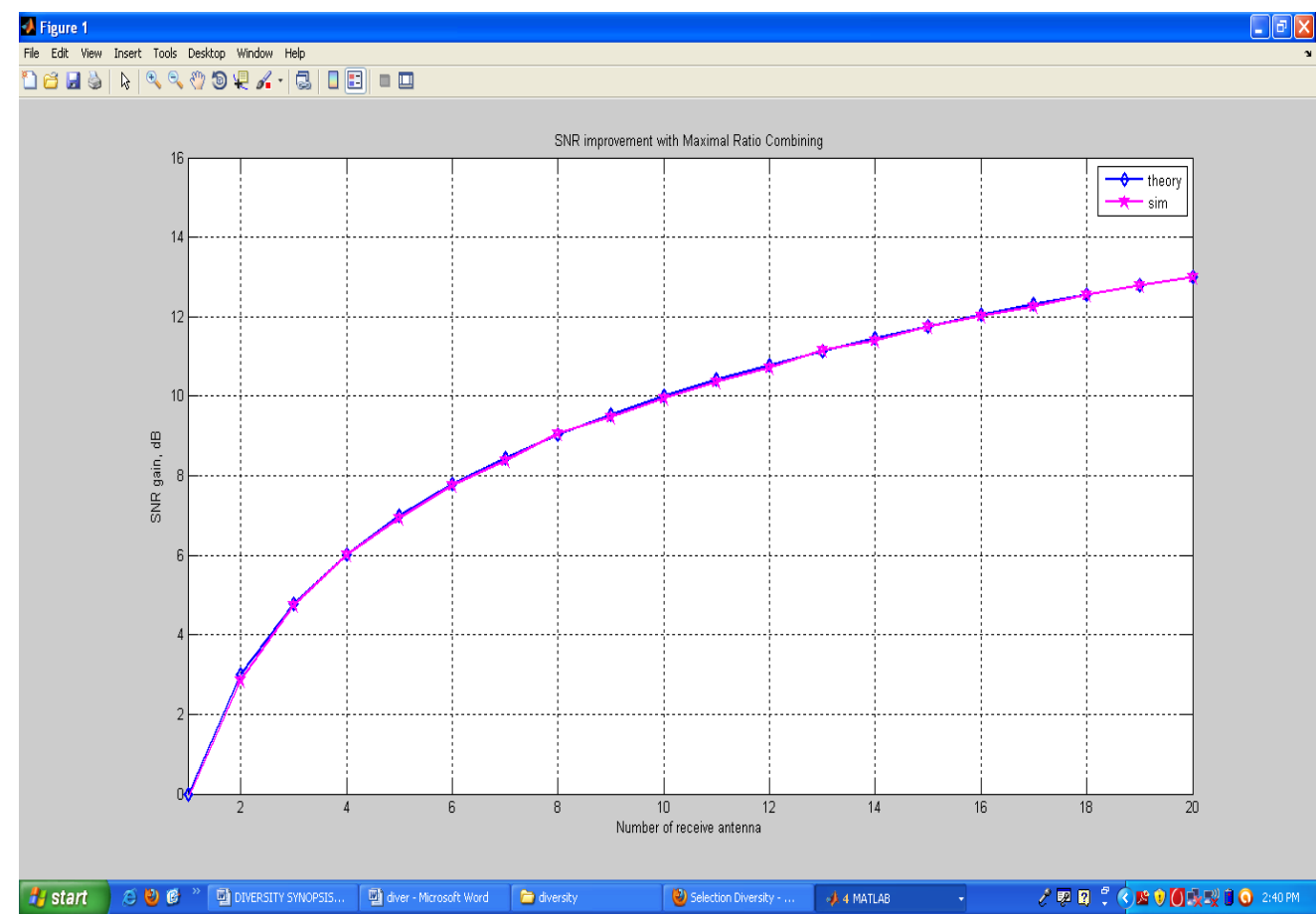

Fig: SNR for Maximal Ratio Combining

\section{V.II.I Result Comparison:}

In the figure below, plot of the SNR with the different no. of antenna for all the three techniques have been shown. This clearly shows that SNR in case of MRC is much than that of Equal Gain and selection diversity. It is also observed that as the number of receiver increases SNR increases gradually. If the no. of antenna is 4 then it can be observed from the below figure the value of signal to noise ratio is more for Maximal Ratio Combining diversity technique as compare to Equal Gain Combining and Selection diversity technique and SNR value for Equal Gain Combining is more than the Selection diversity technique.

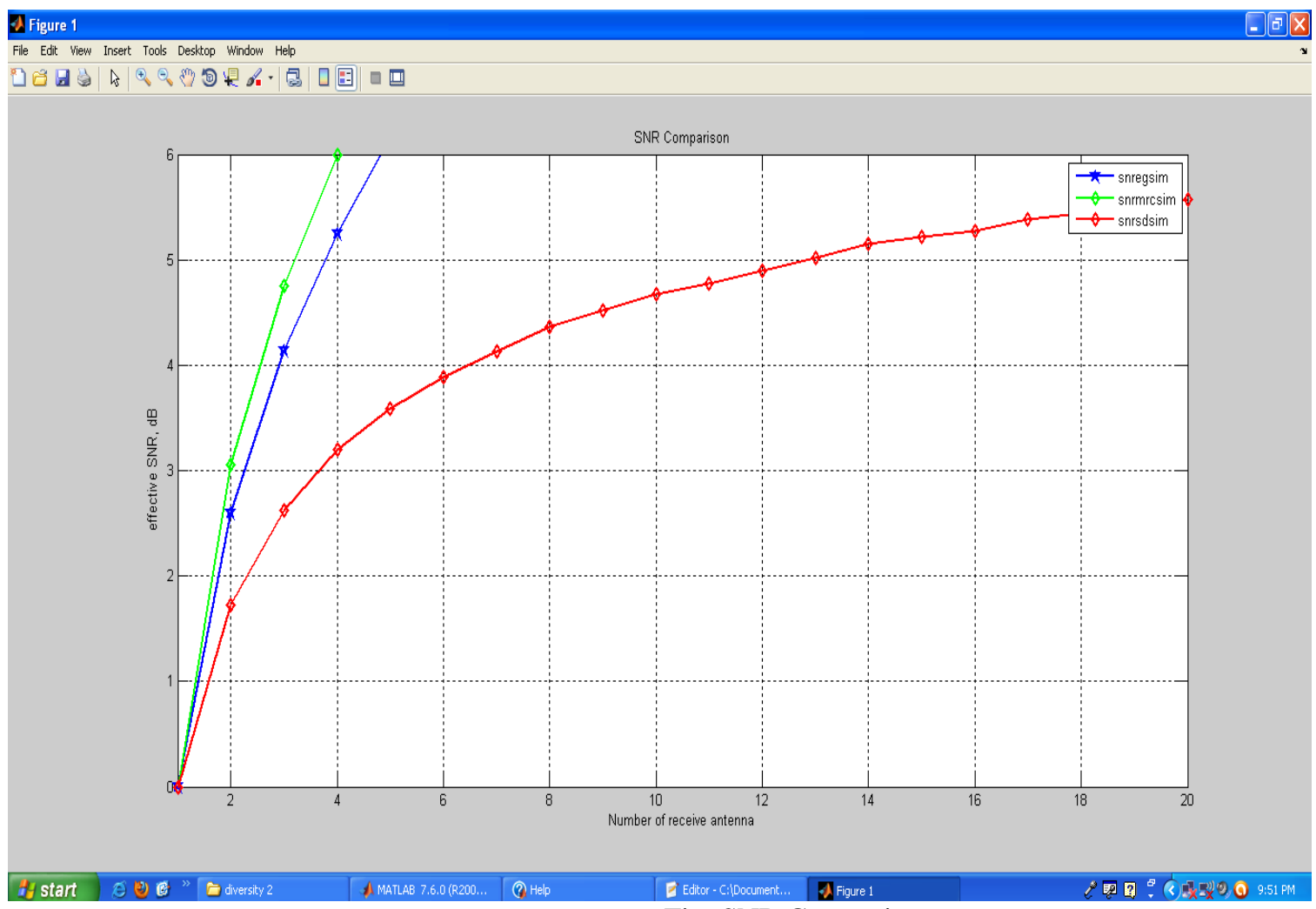

Fig: SNR Comparison 


\section{Conclusion}

As we know for the receiver diversity we have different diversity technique, out of which we used three technique-selection diversity, maximal ratio combining and equal gain combining for our work. BPSK modulation technique and rayleigh fading is used for checking the performance of these technique. We observed that when we calculated the value of SNR with different no. of antenna for these three techniques, selection diversity. For the calculation the bit error rate with respect to the Eb/No then again maximal ratio combining have lesser value as compare to the equal gain combining and selection diversity. So, we can say that the performance of the maximal ratio combining is better as compare to the equal gain combining and selection diversity.

\section{References}

[1] W. C. Jakes, Ed. Microwave Mobile Communications. New York: Wiley, 1974

[2] IEEE TRANSACTIONS ON COMMUNICATIONS, VOL. 16, NO. 8, OCTOBER 1998" A simple Diversity Technique for Wireless Communications" Siavash M. Alamouti, Senior Technical Leader, Alta Business Unit of Cadence Design Systems, Sunnyvale, CA.

[3] IEEE,Abdulkareem Adinoyi, Yijia Fan, Halim Yanikomeroglu, H. Vincent Poor, and Furaih Al-Shaalan "Performance of Selection Relaying and Cooperative Diversity".

[4] IEEE TRANSACTIONS ON COMMUNICATIONS, VOL. 49, NO. 10, OCTOBER 2001" Optimum Selection Diversity for BPSK Signals in Rayleigh Fading Channels" Young Gil Kim and Sang Wu Kim, Senior Menber, IEEE

[5] International Journal of Advanced in Compter Science and Software Engineering "Diversity - A Fading Reduction Technique" Nikita Sachdeva, Deepak Sharma MMU Mullana.

[6] IEEE TRANSACTIONS ON WIRELESS COMMUNICATIONS, 2004 (TO APPEAR) "Performance Analysis of Combined Transmit Selection Diversity and Receive Generalized Selection Combining in Rayleigh Fading channels" Xiaodong Cai, Member, IEEE, and Georgios B. Giannakis, Fellow, IEEE

[7] Vehicular Technology Conference, 2006. VTC-2006 Fall. 2006 IEEE $64^{\text {th }}$ "BER Analysis of BPSK Modulation Over the Weibull Fading Channel with CCI "25-28 Sept. 2006.

[8] D. B. Smith and T. D. Abhayapala are with Wireless Signal Processing, National ICT Australia (NICTA) Locked Bag 8001, Canberra, ACT, 2601 "Maximal Ratio Combining Performance Analysis in Practical Rayleigh Fading Channels".

[9] IEEE David B. Smith and Thushara D. Abhayapala "Maximal Ratio Combining Performance Analysis in Spatially Correlated Rayleigh Fading Channels".

[10] International Journal of Computer Applications (0975 - 8887) Volume 21- No.6, May 2011, "SER Analysis of OFDM System over Rayleigh Fading Channel"'

[11] Communications, IEE Proceedings- "Maximal ratio combining performance analysis in practical Rayleigh fading channels" Oct. 2006 .

[12] Wireless Communications, IEEE Transactions on "Analysis of a two-branch maximal ratio and selection diversity system with unequal SNRs and correlated inputs for a Rayleigh fading channel" Apr 2002

[13] Communications, IEEE Transactions on "A Closed-Form BER Expression for BPSK Using MRC in Correlated CCI and Rayleigh Fading" Dec. 2007

[14] International Journal of Computer Networks \& Communications (IJCNC) Vol.2, No.6, November 2010 "BER PERFORMANCE EVALUATION OF TWO TYPES OF ANTENNA ARRAY-BASED RECEIVERS IN A MULTIPATH CHANNEL”, Rim Haddad1, Ridha Bouallègue.

[15] IEEE TRANSACTIONS ON COMMUNICATIONS, VOL. 53, NO. 8, AUGUST 2005 "Performance Analysis of Predetection EGC in Exponentially Correlated Nakagami-m Fading Channel” P. R. Sahu, Student Member, IEEE, and A. K. Chaturvedi, Senior Member, IEEE

[16] IEEE TRANSACTIONS ON COMMUNICATIONS on "Performance Evaluation of Binary Modulation Schemes with DualDiversity EGC in Rayleigh Fading Environment"

[17] Communications, IEEE Transactions on "Performance of Equal Gain Combining with Quantized Phases in Rayleigh Fading Channels" January 2011

[18] IEEE TRANSACTIONS ON COMMUNICATIONS, Vol. 52 NO. 5, May 2004 on "Performance analysis of Equal Gain Diversity in Nakagami - m Fadin" George K. Karagiannidis, Senior Member, IEEE. 